\title{
Non-Kolmogorov probability models and modified Bell's inequality
}

\author{
Andrei Khrennikov \\ Department of Mathematics, Statistics and Computer Sciences \\ University of Växö, S-35195, Sweden
}

November 5, 2018

\begin{abstract}
We analyse the proof of Bell's inequality and demonstrate that this inequality is related to one particular model of probability theory, namely Kolmogorov measure-theoretical axiomatics, 1933. We found a (numerical) statistical correction to Bell's inequality. Such an additional term $\epsilon_{\phi}$ in the right hand side of Bell's inequality can be considered as a probability invariant of a quantum state $\phi$. This is a measure of nonreproducibility of hidden variables in different runs of experiments. Experiments to verify Bell's inequality can be considered as just experiments to estimate the constant $\epsilon_{\phi}$. It seems that Bell's inequality could not be used as a crucial reason to deny local realism. We consider deterministic as well as stochastic hidden variables models.
\end{abstract}

\section{Introduction}

Experimental violations [1] of Bell's inequality [2] are typically (see, for example, [1],[3]) interpreted in one of two ways: (1) nonlocality: by changing the state of one particle in the EPR pair we change the state of the other particle; (2) death of reality: realism could not be used as the philosophic base of quantum mechanics ('properties' of quantum systems are not objective properties, i.e., the properties of an object). In particular, (2) implies that the statistical interpretation of quantum mechanics (via L. Ballentine, 
[4]) must be denied in favour of the orthodox Copenhagen interpretation. Although such a viewpoint is dominating in the quantum community, there are still some doubts that violations of Bell's inequality must be interpreted in such a way. In particular, many scientists thought (and continue to think) that "Bell's paradox" has purely probabilistic origin, see, for example, [5]. Unfortunately these probabilistic considerations had merely philosophic character. In any case they did not give a new (Bell-like) inequality which has an experimental meaning.

Remark 1.1. The common opinion is that "Bell's paradox" (experimental violations of Bell's inequality) is just a reformulation of the EPR paradox. However, the problem is more complicated: Bell's probabilistic reformulation of the EPR paradox contains some additional assumptions (on probability distribution of hidden variables).

In this note we follow to the general (probabilistic) attitude of [5]. In fact, we generalize ideas of De Baere, [5], on connection of Bell's inequality and "implicit reproducibility" (we arrived to such ideas independently by developing non-Kolmogorov probability formalisms, [6]). However, we found some statistical quantity $\epsilon_{\phi}$ which can be considered as a probability invariant of a quantum state $\phi$. It seems that the standard interpretation of violations of Bell's inequality is a consequence of neglecting of this quantity $\epsilon_{\phi}$.

We analyse Bell's proof and demonstrate that the possibility to derive Bell's inequality depends crucially on the use of a particular probabilistic model, namely the model based on Kolmogorov's axiomatics [7], 1933 (so called measure-theoretical approach to probability). Of course, the Kolmogorov model is the dominating mathematical model for probability theory. Therefore it is not surprising that J. Bell and many others used this approach to probability theory. However, there also exist numerous non-Kolmogorov probabilistic models (which are similar to non-Euclidean geometrical models), see, for example, [8] and Accardi and Gudder in [5]. In particular, I constructed probabilistic models, [6], which describe random phenomena in that the standard law of large numbers is violated: relative frequencies $\nu_{N}=n / N$ have no limit (stabilization), $n \rightarrow \infty$ (numerous examples of such a random behaviour can be found in [6]).

Remark 1.2. We want to underline that in physics the choice of the right probability model is not less important then the choice of the right geometric model. The Kolmogorov model (as well as Euclidean model) could not describe all physical phenomena. 
In this note we analyse "Bell's paradox" on the basis of the assumption that the law of large numbers can be violated for hidden variables: $\nu_{N}(\lambda)$ can fluctuate. From the physical viewpoint this means that different runs of experiments (for example, for correlated particles) can produce different "probability distributions" for hidden variables. In such a situation it would be impossible to define a Kolmogorov probability distribution $\mathbf{P}$ on the set of hidden variables $\Lambda$. Kolmogorov's model could not be applied. We introduce a numerical measure for fluctuations $\epsilon_{\phi}$. It will be shown that "general Bell's inequality" must contain this probability invariant of a quantum state as an additional term.

Finally, we remark that all experimental calculations are, in fact, based not on Kolmogorov model (probability as a measure), but on von Mises, 1919, [9] model (probability as frequency). "Experimental covariation" of two observables, $A, B$, is calculated as a sequence mean value: $\langle A, B\rangle_{\mathrm{fr}}=$ $(1 / N) \sum_{i=1}^{N} A_{i} B_{i}$, where $x=\left(A_{1}, A_{2}, \ldots, A_{N}\right)$ and $y=\left(B_{1}, B_{2}, \ldots, B_{N}\right)$ are random sequences (collectives) generated by measurements of $A$ and $B$. Hence, in fact, $<A, B>_{\mathrm{fr}}$ depends on $x$ and $y:<A, B>_{\mathrm{fr}}=<A, B>_{x y}$. However, J. Bell supposed that there exists a Kolmogorov probability distribution $\mathbf{P}$ on the set of hidden variables $\Lambda$ and all covariations can be written as mean values with respect to this unique measure: $<A, B>_{\mathrm{Bell} / \mathrm{Kol}}=$ $\int_{\Lambda} A(\lambda) B(\lambda) d \mathbf{P}(\lambda)$. This (rather strong) assumption statistical postulate has never been verified experimentally.

\section{Bell's proof}

We reproduce the proof of Bell's inequality. Let $\mathcal{P}=(\Omega, \mathcal{F}, \mathbf{P})$ be a Kolmogorov probability space: $\Omega$ is a space of elementary events, $\mathcal{F}$ is an algebra of events, $\mathbf{P}$ probability measure.

Theorem 1. Let $A, B, C= \pm 1$ be random variables on $\mathcal{P}$. Then Bell's inequality

$$
|<A, B>-<C, B>| \leq 1-<A, C>
$$

holds true.

Proof. Set $\Delta=<A, B>-<C, B>$. By linearity of Lebesgue integral we obtain

$$
\Delta=\int_{\Omega} A(\omega) B(\omega) d \mathbf{P}(\omega)-\int_{\Omega} C(\omega) B(\omega) d \mathbf{P}(\omega)=\int_{\Omega}[A(\omega)-C(\omega)] B(\omega) d \mathbf{P}(\omega) .
$$


As $A(\omega)^{2}=1$,

$$
|\Delta|=\left|\int_{\Omega}[1-A(\omega) C(\omega)] A(\omega) B(\omega) d \mathbf{P}(\omega)\right| \leq \int_{\Omega}[1-A(\omega) C(\omega)] d \mathbf{P}(\omega) .
$$

Of course, this is the rigorous mathematical proof of (11) for Kolmogorov probabilities. However, abstractness of Kolmogorov's probability model induces serious problems, if we do not control carefully dependence of probabilities on corresponding statistical ensembles of physical systems. Bell did not control this dependence. In fact, the symbol $\mathbf{P}$ of probability which is used in the proof must be regarded to different statistical ensembles.

\section{Fluctuating distributions of hidden variables}

To simplify our considerations, we suppose that the set of hidden variables is finite: $\Lambda=\left\{\lambda_{1}, \ldots, \lambda_{M}\right\}$. For each physical observable $U$, the value $\lambda$ of hidden variables determines the value $U=U(\lambda)$. Let $U$ and $V$ be physical observables, $U, V= \pm 1$. We start with the consideration of the frequency (experimental) covariation $\langle U, V\rangle_{x_{U V}}$ with respect to a random sequence $x_{U V}=\left(x_{1}, x_{2}, \ldots, x_{N}, \ldots\right)$, where $x_{i}=\left(u_{i}, v_{i}\right)$, which is induced by measurements of the pair $(U, V)$. The $x_{U V}$ is obtained by measurements for an ensemble $S_{U V}$ of physical systems (for example, pairs of correlated quantum particles). Our aim is to represent experimental covariation $<U, V\rangle_{x_{U V}}$ as ensemble covariation $\langle U, V\rangle_{S_{U V}}$. Then we shall demonstrate that in the general case it is impossible to perform for ensemble covariations Bell's calculations which have been performed for Kolmogorov covariations. Let $S_{U V}=\left\{d_{1}, \ldots, d_{N}\right\}$, where $i$ th measurement is performed for the system $d_{i}$. Define a function $i \rightarrow \lambda(i)$, the value of hidden variables for $d_{i}$. We set

$n_{k}\left(S_{U V}\right)=\left|\left\{d_{i} \in S_{U V}: \lambda(i)=\lambda_{k}\right\}\right|$ and $\mathbf{p}_{k}^{U V}=\mathbf{P}_{S_{U V}}\left(\lambda=\lambda_{k}\right)=\frac{n_{k}\left(S_{U V}\right)}{N}$. These are probabilities of hidden variables $\lambda_{k}, k=1,2, \ldots, M$, in the statistical ensemble $S_{U V}$. We have $<U, V>_{x_{U V}}=\frac{1}{N} \sum_{i=1}^{N} U(\lambda(i)) V(\lambda(i))=$ $\sum_{k=1}^{M} \mathbf{p}_{k}^{U V} u_{k} v_{k}=<U, V>_{S_{U V}}$, where $u_{k}=U\left(\lambda_{k}\right), v_{k}=V\left(\lambda_{k}\right)$. Thus

$$
\begin{gathered}
\Delta=<A, B>_{x_{A B}}-<C, B>_{x_{C B}} \\
=<A, B>_{S_{A B}}-<C, B>_{S_{C B}}=\sum_{k}\left(\mathbf{p}_{k}^{A B} a_{k}-\mathbf{p}_{k}^{C B} c_{k}\right) b_{k}
\end{gathered}
$$

and

$$
<A, C>_{x_{A C}}=<A, C>_{S_{A C}}=\sum_{k} \mathbf{p}_{k}^{A C} a_{k} c_{k}
$$


We now suppose that probabilities of $\lambda_{k}$ do not depend on statistical ensembles:

$$
\mathbf{p}_{k}=\mathbf{p}_{k}^{A B}=\mathbf{p}_{k}^{C B}=\mathbf{p}_{k}^{A C}
$$

(later we shall modify this condition to obtain statistical coincidence of probabilities, instead of the precise coincidence). Hence $\Delta=\sum_{k=1}^{M} \mathbf{p}_{k}\left(a_{k}-\right.$ $\left.c_{k}\right) b_{k}$ and $\quad<A, C>_{x_{A C}}=\sum_{k=1}^{M} \mathbf{p}_{k} a_{k} c_{k}$. We can now apply Theorem 1 for the discrete probability distribution $\left\{\mathbf{p}_{k}\right\}_{k=1}^{M}$ and obtain Bell's inequality.

However, if condition (4) does not hold true, then equality (2) and, as a consequence, Bell's inequality can be violated. The violation of condition (4) is the exhibition of unstable statistical structure on the level of hidden variables. Condition (4) is equivalent to a condition of implicit reproducibility which was discussed by De Baere [5].

Remark 3.1. ( $p$-adic probability models, negative probabilities and Bell's inequality). All our considerations were based on the statistical stabilization with respect to the real metric. In [6] we considered the statistical stabilization with respect to a $p$-adic metric. The field of $p$-adic numbers $\mathbf{Q}_{p}$, where $p>1$ is a prime number, can be constructed (as the field of real numbers $\mathbf{R})$ as a completion of the field of rational numbers $\mathbf{Q}$. The $p$-adic metric differs strongly from the real one. As for finite ensembles $S$, ensemble probabilities $\mathbf{P}_{S}(a)=\frac{n(a)}{N}$ are rational numbers, we can study their behaviour not only with respect to the real metric on $\mathbf{Q}$, but also with respect to the $p$-adic metric. $p$-adic probability theory gives numerous examples of ensemble probabilities fluctuating in the real metric and stabilizing in the $p$-adic metric. However, the $p$-adic stabilization of probabilities does not imply the possibility to repeat Bell's proof for $p$-adic probabilities: these probabilities may be negative rational numbers, see [6] (compare with Muckenheim, [5]).

\section{Measure of statistical deviation between runs of an experi- ment}

We introduce now a statistical analogue of the precise coincidence of ensemble probabilities for hidden variables. Let $\mathcal{E}_{1}, \mathcal{E}_{2}$ be two ensembles of physical systems and let $\pi$ be a property of elements of these ensembles. The $\pi$ has values $\left(\alpha_{1}, \ldots, \alpha_{m}\right)$. We define

$$
\delta_{\pi}\left(\mathcal{E}_{1}, \mathcal{E}_{2}\right)=\sum_{i=1}^{M}\left|\mathbf{P}_{\mathcal{E}_{1}}\left(\alpha_{i}\right)-\mathbf{P}_{\mathcal{E}_{2}}\left(\alpha_{i}\right)\right|,
$$


where $\mathbf{P}_{\mathcal{E}}\left(\alpha_{i}\right)=\frac{\left|\left\{d \in \mathcal{E}: \pi(d)=\alpha_{i}\right\}\right|}{|\mathcal{E}|}$ are ensemble probabilities. We remark that the function $\delta=\delta_{\pi}$ is a pseudometric on the set of all ensembles which elements have the property $\pi:$ 1) $\left.\left.\delta\left(\mathcal{E}_{1}, \mathcal{E}_{2}\right) \geq 0 ; 2\right) \delta\left(\mathcal{E}_{1}, \mathcal{E}_{2}\right)=\delta\left(\mathcal{E}_{2}, \mathcal{E}_{1}\right) ; 3\right) \delta\left(\mathcal{E}_{1}, \mathcal{E}_{2}\right) \leq$ $\delta\left(\mathcal{E}_{1}, \mathcal{E}_{3}\right)+\delta\left(\mathcal{E}_{3}, \mathcal{E}_{2}\right)$. In our model we set $\pi=\lambda$, hidden variables. The precise reproducibility of the probability distribution of hidden variables (雨) can be written as

$$
\delta\left(S_{A B}, S_{C B}\right)=\delta\left(S_{A B}, S_{A C}\right)=0,
$$

where $\delta=\delta_{\lambda}$. Of course, we need not use such a precise coincidence in probabilistic considerations. Let $\phi$ be a quantum state. Denote by the symbol $T_{\phi}$ the set of all statistical ensembles $\mathcal{E}$ which correspond to $\phi$ (can be obtained with the aid of some preparation procedure corresponding to $\phi$ ). Set

$$
\epsilon_{\phi}=\sup \left\{\delta\left(\mathcal{E}_{1}, \mathcal{E}_{2}\right): \mathcal{E}_{1}, \mathcal{E}_{2} \in T_{\phi}\right\}
$$

Theorem 2. ("General Bell's inequality") Let $\phi$ be a quantum state and let $A, B, C$ be physical observables such that pairs of observables $(A, B),(C, B)$ and $(A, C)$ can be measured. Then inequality

$$
|<A, B>-<C, B>| \leq\left(1+2 \epsilon_{\phi}\right)-<A, C>
$$

holds true.

Proof. We have

$$
\begin{gathered}
|\Delta|=\left|<A, B>_{x_{A B}}-<C, B>_{x_{C B}}\right| \\
\leq\left|\sum_{k=1}^{M} \mathbf{p}_{k}^{A B}\left(a_{k}-c_{k}\right) b_{k}\right|+\left|\sum_{k=1}^{M}\left(\mathbf{p}_{k}^{A B}-\mathbf{p}_{k}^{C B}\right) c_{k} b_{k}\right| \\
\leq \epsilon_{\phi}+\sum_{k=1}^{M} \mathbf{p}_{k}^{A B}\left|a_{k} b_{k}\right|\left(1-a_{k} c_{k}\right) \leq\left(1+\epsilon_{\phi}\right)-<A, C>_{S_{A C}}+\sum_{k=1}^{M}\left|\mathbf{p}_{k}^{A C}-\mathbf{p}_{k}^{A B}\right|\left|a_{k} c_{k}\right| \\
\leq\left(1+2 \epsilon_{\phi}\right)-<A, C>_{S_{A C}} .
\end{gathered}
$$

We use the index $N$ to denote the cardinality of a statistical ensemble. If probabilities $\mathbf{P}_{S_{U V}^{N}}\left(\lambda_{k}\right)$ stabilize when $N \rightarrow \infty$,

$$
\lim _{N \rightarrow \infty} \mathbf{P}_{S_{U V}^{N}}\left(\lambda_{k}\right)=\mathbf{P}\left(\lambda_{k}\right)
$$


then $\epsilon_{\phi}^{N} \rightarrow 0, N \rightarrow \infty$. This imply precise Bell's inequality (11).

Experiments to verify Bell's inequality can be considered as experiments to estimate the probability invariant $\epsilon_{\phi}$ for some class of quantum states. It seems that the only lesson of these experiments is that there exist quantum states $\phi$ which have nonzero probability invariant $\epsilon_{\phi}$. It may be that physical reality is nonlocal. It may be that it is even nonreal. However, it seems that Bell's arguments did not imply neither nonlocality nor nonreality.

\section{Stochastic hidden variables model}

In this section it is supposed that the result of a measurement depends not only on the value $\lambda$ of hidden variables, but also on the state $\omega^{U}$ of an equipment $\mathcal{M}_{U}$ which is used for measuring of $U$. This the empiricists (contextualistic realists) interpretation of quantum mechanics, see, for example, W. De Muynck, W. De Baere, H. Marten, Ref. [5].

A measurement device $\mathcal{M}_{U}$ is a complex macroscopic system which state depends on the huge number of fluctuating parameters. Denote the ensemble

of all possible states of $\mathcal{M}_{U}$ by the symbol $\Sigma_{U}: \Sigma_{U}=\left\{\omega_{1}^{U}, \ldots, \omega_{L_{U}}^{U}\right\}$. The final value $U_{f}$ of an observable $u$ depends on both $\lambda$ and $\omega$ :

$$
u=U(\omega, \lambda) .
$$

We call such a model stochastic hidden variables model. Our model of stochastic hidden variables differs from the standard one, see section 6 . The latter model is strongly connected with Kolmogorov's probability model (existence of the probability distribution of hidden variables $\mathbf{P}(\lambda)$ and conditional probabilities $\mathbf{P}(U, \lambda)$ is postulated).

Let $U$ and $V$ be physical observables, $U, V= \pm 1$. We start again with the consideration of the frequency covariation $\langle U, V\rangle_{x_{U V}}$ with respect to a collective $x_{U V}$ induced by the measurement of the pair $(U, V)$. The $x_{U V}$ is obtained by measurements for an ensemble $S_{U V}$ of physical systems. Our aim is again to represent the experimental covariation $\langle U, V\rangle_{x_{U V}}$ as ensemble covariation $\left\langle U, V>_{S_{U V}}\right.$. Then we shall demonstrate that in the general case it is impossible to perform for ensemble covariations Bell's calculations, (2) - (3).

Let $S_{U V}=\left\{d_{1}, \ldots, d_{N}\right\}$, where $i$ th measurement is performed for the system $d_{i}$. Define functions $i \rightarrow \lambda(i)$ (the same function as above) and $i \rightarrow \omega^{U}(i), i \rightarrow \omega^{V}(i)$, states of apparatus $\mathcal{M}_{U}$ and $\mathcal{M}_{V}$, respectively, at 
the instances, $t_{i}^{U}$ and $t_{i}^{V}$, of measurements of $U$ and $V$ for $i$ th system. We have

$$
<U, V>_{x_{U V}}=\frac{1}{N} \sum_{i=1}^{N} U\left(\omega^{U}(i), \lambda(i)\right) V\left(\omega^{V}(i), \lambda(i)\right) .
$$

Set $D_{k s}^{U}=\left\{i: \lambda(i)=\lambda_{k}, \omega^{U}(i)=\omega_{s}^{U}\right\}$ and $D_{k s}^{V}=\left\{i: \lambda(i)=\lambda_{k}, \omega^{V}(i)=\right.$ $\left.\omega_{s}^{V}\right\}, 1 \leq k \leq M, 1 \leq s \leq L_{U}, 1 \leq q \leq L_{V}$. Set $l_{k s q}^{U V}=\left|D_{k s}^{U} \cap D_{k q}^{V}\right|$. It is evident that

$$
\sum_{k=1}^{M} \sum_{s=1}^{L_{U}} \sum_{q=1}^{L_{V}} l_{k s q}^{U V}=N
$$

Hence

$$
<U, V>_{x_{U V}}=\frac{1}{N} \sum_{k s q} l_{k s q}^{U V} u_{k s} v_{k q}
$$

where $u_{k s}=U\left(\omega_{s}^{U}, \lambda_{k}\right), v_{k q}=V\left(\omega_{q}^{V}, \lambda_{k}\right)$. We show that $\left\langle U, V>_{x_{U V}}\right.$ can be represented as ensemble covariation for an appropriative ensemble of physical systems and states of measurement devices. However, a choice of such an ensemble is rather delicate problem.

First we note that $<U, V>_{x_{U V}} \neq<U, V>_{\Lambda \times \Sigma_{A} \times \Sigma_{B}}$ (compare with section $6)$. For the latter covariation, we have

$$
<U, V>_{\Lambda \times \Sigma_{A} \times \Sigma_{B}}=\frac{1}{M L_{A} L_{B}} \sum_{k=1}^{M} \sum_{s=1}^{L_{U}} \sum_{q=1}^{L_{V}} u_{k s} v_{k q}
$$

and in general $\mathbf{P}_{\Lambda \times \Sigma_{A} \times \Sigma_{B}}\left(\lambda=\lambda_{k}, \omega^{U}=\omega_{s}^{U}, \omega^{V}=\omega_{q}^{V}\right)=\frac{1}{M L_{A} L_{B}} \neq \frac{l_{k s q}}{N}$ even approximately for $M, N, L_{A}, L_{B} \rightarrow \infty$.

It is also evident that $\left\langle U, V>_{x_{U V}} \neq<U, V>_{S_{U V}}\right.$. The latter covariation is simply not well defined, because the 'properties' $\omega^{U}(i)=\omega_{s}^{U}, \omega^{V}(i)=\omega_{q}^{V}$ are not objective properties of elements of the ensemble $S_{U V}$. These "properties' are determined by fluctuations of parameters in the apparatus $\mathcal{M}_{U}$ and $\mathcal{M}_{V}$.

To find the right ensemble, we have to introduce two new ensembles, namely, ensembles of states of the apparatus $\mathcal{M}_{U}$ and $\mathcal{M}_{V}$ (in the process of measurements for the ensemble of physical systems $\left.S_{U V}\right)$ :

$$
S_{\mathcal{M}_{U}}=\left\{\alpha_{1}^{U}, \ldots, \alpha_{N}^{U}\right\}, \alpha_{j}^{U} \in \Sigma_{U}, S_{\mathcal{M}_{V}}=\left\{\alpha_{1}^{V}, \ldots, \alpha_{N}^{V}\right\}, \alpha_{j}^{V} \in \Sigma_{V},
$$


where $\alpha_{i}^{U}=\omega^{U}(i), \alpha_{i}^{V}=\omega^{V}(i)$ are states of $\mathcal{M}_{U}$ and $\mathcal{M}_{V}$ at the instances of $i$ th measurements. We set

$$
\mathbf{S}_{U V}=\operatorname{diag}\left(\mathrm{S}_{\mathrm{UV}} \times \mathrm{S}_{\mathcal{M}_{\mathrm{U}}} \times \mathrm{S}_{\mathcal{M}_{\mathrm{V}}}\right)=\left\{\mathrm{D}_{1}, \ldots, \mathrm{D}_{\mathrm{N}}\right\}, \quad \mathrm{D}_{\mathrm{j}}=\left(\mathrm{d}_{\mathrm{j}}, \alpha_{\mathrm{j}}^{\mathrm{U}}, \alpha_{\mathrm{j}}^{\mathrm{V}}\right) .
$$

Then $\pi\left(D_{j}\right)=\left(\lambda(j), \omega^{U}(j), \omega^{V}(j)\right)$ is an objective property of elements of the ensemble $\mathbf{S}_{U V}$ and

$$
<U, V>_{x_{U V}}=<U, V>_{\mathbf{s}_{U V}}=\frac{1}{N} \sum_{i=1}^{N} U\left(\omega^{U}(i), \lambda(i)\right) V\left(\omega^{V}(i), \lambda(i)\right) .
$$

We set

$$
\begin{aligned}
& \mathbf{p}_{k s q}^{U V}=\mathbf{P}_{\mathbf{S}_{U V}}\left(D_{j}: \pi\left(D_{j}\right)=\left(\lambda_{k}, \omega_{s}^{U}, \omega_{s}^{V}\right)\right) \\
& =\frac{\left|\left\{D_{j} \in \mathbf{S}_{U V}: \pi\left(D_{j}\right)=\left(\lambda_{k}, \omega_{s}^{U}, \omega_{s}^{V}\right)\right\}\right|}{\left|\mathbf{S}_{U V}\right|} .
\end{aligned}
$$

Hence we obtained that

$$
<U, V>_{x_{U V}}=<U, V>_{\mathbf{s}_{U V}}=\sum_{k s q} \mathbf{p}_{k s q}^{U V} u_{k s} v_{k q}
$$

Thus in the general case we have

$$
\begin{aligned}
& \Delta=<A, B>_{x_{A B}}-<C, B>_{x_{C B}}=<A, B>_{\mathbf{s}_{A B}}-<C, B>_{\mathbf{S}_{C B}} \\
& =\sum_{k s q} \mathbf{p}_{k s q}^{A B} a_{s k} b_{k q}-\sum_{k s q} \mathbf{p}_{k s q}^{C B} c_{k s} b_{k q}
\end{aligned}
$$

and

$$
<A, C>_{x_{A C}}=<A, C>_{\mathbf{s}_{A C}}=\sum_{k s q} \mathbf{p}_{k s q}^{A C} a_{k s} c_{k q} .
$$

We suppose now that probabilities $\mathbf{p}_{k s q}^{U V}$ do not depend on ensembles:

$$
\mathbf{p}_{k s q}=\mathbf{p}_{k s q}^{A B}=\mathbf{p}_{k s q}^{C B}=\mathbf{p}_{k s q}^{A C}
$$

In particular, we suppose that all measurement devices have the same set of states (of parameters):

$$
\Sigma=\Sigma_{A}=\Sigma_{B}=\Sigma_{C}\left(\text { and } L=L_{A}=L_{B}=L_{C}\right) .
$$


Then we obtain

$$
\Delta=\sum_{k s q} \mathbf{p}_{k s q}\left(a_{k s}-c_{k s}\right) b_{k q}
$$

However, we could not repeat trick (3) of the proof of Bell's inequality. The equality $a_{k s}^{2}=1$ does not give the possibility to proceed the proof. Of course, we have

$$
\begin{aligned}
& |\Delta|=\left|\sum_{k s q} \mathbf{p}_{k s q}\left(a_{k s}-a_{k s}^{2} c_{k s}\right) b_{k q}\right| \leq \sum_{k s q} \mathbf{p}_{k s q}\left|a_{k s} b_{k q}\right|\left(1-a_{k s} c_{k s}\right) \\
& \leq 1-\sum_{k s q} \mathbf{p}_{k s q} a_{k s} c_{k s} .
\end{aligned}
$$

But in general $\sum_{k s q} \mathbf{p}_{k s q} a_{k s} c_{k s}$ is not larger than $<A, C>_{x_{A C}}=\sum_{k s q} \mathbf{p}_{k s q} a_{k s} c_{k q}$.

Therefore, if we keep to empiricism, then even stability condition (6) (for combined ensembles of physical systems and states of measurement apparatus) does not imply Bell's inequality. A new source of violation of Bell's inequality is the inconsistency of random fluctuations for two measurement devices $\mathcal{M}_{U}$ and $\mathcal{M}_{V}$. In general $\omega^{U}(i) \neq \omega^{V}(i)$.

Suppose that it could be possible to control states of $\mathcal{M}_{U}$ and $\mathcal{M}_{V}$ and choose $\omega$ for $\mathcal{M}_{U}$ and $\mathcal{M}_{V}$ in the consistence way:

$$
\omega=\omega^{U}(i)=\omega^{V}(i) .
$$

Then the ensemble $\mathbf{S}_{U V}$ would contain only triples of the form $\left(\lambda_{k}, \omega_{s}, \omega_{s}\right)$ and

$$
\mathbf{p}_{k s q}^{U V}=\mathbf{P}_{\mathbf{S}_{U V}}\left(\lambda_{k}, \omega_{s}^{U}, \omega_{q}^{V}\right)=0, s \neq q .
$$

In such a case we obtain covariations:

$$
<U, V>_{\text {Ideal }}=\frac{1}{N} \sum_{i=1}^{N} U\left(\omega^{U}(i), \lambda(i)\right) V\left(\omega^{V}(i), \lambda(i)\right)=\sum_{k s} \mathbf{p}_{k s}^{U V} u_{k s} v_{k s},
$$

where $\mathbf{p}_{k s}^{U V}=\mathbf{p}_{k s s}^{U V}$. If we also suppose the validity of (6), we obtain

$$
\begin{gathered}
\left|\Delta_{\text {Ideal }}\right|=\left|\sum_{k s} \mathbf{p}_{k s}\left(a_{k s}-c_{k s}\right) b_{k s}\right| \\
\leq 1-\sum_{k s} \mathbf{p}_{k s} a_{k s} c_{k s}=1-<A, C>_{\text {Ideal }} .
\end{gathered}
$$

However, ideal covariations have no direct connection to experimental frequency covariations. 
Nevertheless, we can formulate the following mathematical theorem:

Theorem 3. Let statistical ensembles (physical systems/measurement apparatus) satisfy conditions (6) and (8). Then Bell's inequality (14) holds true for covariations with respect to these ensembles.

Therefore, to obtain Bell's inequality in the empiricists framework, we have to suppose: (1) statistical repeatability of ensemble distribution of hidden variables $\lambda$ in ensembles which are used for measurements; (3) statistical repeatability of fluctuations of states $\omega$ in ensembles of an equipment; (3) consistency of fluctuations of all measurement devices.

If the reader even deny the possibility of violations of (1) or (2), he must agree that condition (3) seems to be nonphysical: we could never control fluctuations of the huge number of parameters in the equipment.

Instead of precise coincidence (6), it is possible to consider (under the assumption (7)) the statistical coincidence based on the quantity:

$$
\delta\left(\mathbf{S}_{A B}, \mathbf{S}_{C B}\right)=\sum_{k=1}^{M} \sum_{s=1}^{L} \sum_{q=1}^{L}\left|\mathbf{p}_{k s q}^{A B}-\mathbf{p}_{k s q}^{C B}\right| .
$$

Here $\delta=\delta_{\pi}$ for the property $\pi(i)=\left(\lambda(i), \omega^{U}(i), \omega^{V}(i)\right)$. We remark that condition (6) of the precise coincidence can be written as

$$
\delta\left(\mathbf{S}_{A B}, \mathbf{S}_{C B}\right)=0
$$

for every two pairs of observable $(A, B)$ and $(C, B)$. We also introduce a new quantity which is a statistical measure of inconsistency of ensembles $S_{\mathcal{M}_{U}}$ and $S_{\mathcal{M}_{V}}$ :

$$
\sigma\left(\mathbf{S}_{U V}\right)=\sum_{s \neq q} \mathbf{P}_{\mathbf{S}_{U V}}\left(\omega^{U}=\omega_{s}, \omega^{V}=\omega_{q}\right)=\sum_{k} \sum_{s \neq q} \mathbf{p}_{k s q}^{U V} .
$$

Condition (8) of the precise consistency for states of $\mathcal{M}_{U}$ and $\mathcal{M}_{V}$ can be written in the form:

$$
\sigma\left(\mathbf{S}_{U V}\right)=0 .
$$

Theorem 4. Let statistical ensembles (physical systems/measurement apparatus) satisfy conditions:

$$
\delta\left(\mathbf{S}_{A B}, \mathbf{S}_{C B}\right), \delta\left(\mathbf{S}_{A B}, \mathbf{S}_{A C}\right) \leq \epsilon \text { and } \sigma\left(\mathbf{S}_{A B}\right), \sigma\left(\mathbf{S}_{C B}\right), \sigma\left(\mathbf{S}_{A C}\right) \leq \epsilon^{\prime} .
$$


Then inequality

$$
\left|<A, B>\mathbf{S}_{A B}-<C, B>_{\mathbf{S}_{C B}}\right| \leq\left(1+2 \epsilon+3 \epsilon^{\prime}\right)-<A, C>_{\mathbf{S}_{A C}}
$$

holds true.

Proof. We have

$$
\begin{gathered}
|\Delta| \leq \epsilon+\left|\sum_{k s q} \mathbf{p}_{k s q}^{A B}\left(a_{k s}-c_{k s}\right) b_{k q}\right| \\
\leq \epsilon+2 \epsilon^{\prime}+\sum_{k s} \mathbf{p}_{k s}^{A B}\left|\left(a_{k s}-c_{k s}\right) b_{k s}\right| \leq \epsilon+2 \epsilon^{\prime}+\sum_{k s} \mathbf{p}_{k s}^{A B}\left(1-a_{k s} c_{k s}\right) \\
\leq \epsilon+4 \epsilon^{\prime}+\sum_{k s q} \mathbf{p}_{k s q}^{A B}\left(1-a_{k s} c_{k q}\right) \leq\left(1+2 \epsilon+4 \epsilon^{\prime}\right)-\sum_{k s q} \mathbf{p}_{k s q}^{A C} a_{k s} c_{k q} .
\end{gathered}
$$

We remark again that experiments to test Bell's inequality can be interpreted as just experiments to find an estimate for a constant $C=2 \epsilon+4 \epsilon^{\prime}$. From this point of view the only result of these experiments is that $C$ is essentially larger that zero. However, such a results could be expected: it would be rather strange if measures of statistical deviations $\delta$ and $\sigma$ would be equal to zero despite of fluctuations of parameters of measuring devices.

\section{Probability distributions in stochastic hidden variables mod-} els.

Typically stochastic hidden variables models are defined as models with probabilities $(\epsilon= \pm 1)$

$$
\mathbf{P}(U=\epsilon)=\int_{\Lambda} \mathbf{P}(U=\epsilon / \lambda) d \rho(\lambda),
$$

where $\rho(\lambda)$ is the probability distribution of hidden variables and $\mathbf{P}(U=\epsilon / \lambda)$ is the conditional probability to measure the value $U=\epsilon$ for the quantum system having the hidden state $\lambda$, see, for example, [10].

Then (see Ref. [11]) the joint probability distribution can be defined (at least mathematically) as

$\mathbf{P}\left(U_{1}=\epsilon_{1}, U_{2}=\epsilon_{2}, U_{3}=\epsilon_{3}\right)=\int_{\Lambda} \mathbf{P}\left(U_{1}=\epsilon_{1} / \lambda\right) \mathbf{P}\left(U_{2}=\epsilon_{2} / \lambda\right) \mathbf{P}\left(U_{3}=\epsilon_{3} / \lambda\right) d \rho(\lambda)$. 
In fact, to derive Bell's inequality in the Kolmogorov framework, it is sufficient to use the existence (on the mathematical level) of the joint probability distribution (10). However, considerations in the framework of the ensemble probability theory demonstrated that 'probabilities' (9) has no physical meaning. These are probabilities with respect to the ensemble $\Lambda \times \Sigma_{U}$. However, physical probabilities are probabilities with respect to the ensemble $\mathbf{S}_{U}=\operatorname{diag}\left(\mathrm{S}_{\mathrm{U}} \times \mathrm{S}_{\mathcal{M}_{\mathrm{U}}}\right)$, where $S_{U}=\left\{d_{1}, \ldots, d_{N}\right\}$ is the ensemble of quantum system used in the measurement. We note that physical arguments against existing of representation (9) were presented by W. De Muynck, W. De Baere, H. Marten in Ref. [5]. I think that results of this paper can strongly improve their considerations. We hope that our numerical description of nonexistence of Kolmogorov probabilities could essentially clarify the problem.

\section{Other probabilistic models which do not contradict to local realism.}

L. Accardi in Ref. [5] used non-Kolmogorov model without Bayes' formula to eliminate Bell's inequality from considerations related to spin's model. Recently he developed a new model which gives an explanation of violations of Bell's inequality, see Ref. [12]. In fact, to get "physical Bell's inequality" we have to consider in Theorem 1 indexed random variables $U^{1}$ and $U^{2}$ corresponding to correlated particles, 1 and 2. "Physical Bell's inequality" can be obtained only on the basis of the implicit anticorrelation: $U^{1}=-U^{2}$. Accardi discussed the role of this condition in Bell's arguments.

I. Pitowsky in Ref. [5] discussed the possibility that some nonmeasurable sets can be physical events, i.e, some physical observables may be nonmeasurable. There is no Bell's inequality in this approach. Thus there is no problem with violations of Bell's inequality. This model is consistent with known polarization phenomena and the existence of macroscopic magnetism. He also proposed a thought experiment which indicates a deviation from the predictions of quantum mechanics. We note that already A. N. Kolmogorov discussed 'generalized probabilities' on the algebra of all subsets of $\Omega$. Mathematicians, in particular applied mathematicians, where reluctant to take nonmeasurable sets seriously. As a result there was no mathematical theory that relates nonmeasurable distributions with relative frequencies. Such an extension of probability theory was created by I. Pitowsky and then strongly mathematically improved by S.P. Gudder in Ref. [5]. He introduced the concept of a probability manifold $M$. The global properties of $M$ inherited from 
its local structure were then considered. It was shown that a deterministic spin model due to Pitowski falls within this general framework. Finally, Gudder constructed a phase-space model for nonrelativistic quantum mechanics. These two models give the same global description as conventional quantum mechanics. However, they also give a local descriptions which is not possible in conventional quantum mechanics.

\section{ACKNOWLEDGMENTS}

This paper was completed during the visit to Moscow State University

on the basis of the grant of the Royal Academy of Science (Sweden) for the collaboration with the former Soviet Union.

\section{References}

[1] J.F. Clauser , A. Shimony, Rep. Progr.Phys., 41 1881-1901 (1978). A. Aspect, J. Dalibard, G. Roger, Phys. Rev. Lett., 49, 1804-1807 (1982); D. Home, F. Selleri, Nuovo Cim. Rivista, 14, 2-176 (1991).

[2] J.S. Bell, Rev. Mod. Phys., 38, 447-452 (1966).

[3] B. d'Espagnat, Veiled Reality. An anlysis of present-day quantum mechanical concepts. Addison-Wesley(1995).

[4] L. E. Ballentine, Rev. Mod. Phys., 42, 358-381 (1970).

[5] L. De Broglie, La Physique Quantique Restera-t-elle Indeterministe? Gauthier-Villars, Paris (1953); Lochak G., De Broglie's initial conception of de Broglie waves. The wave-particle dualism. A tribute to Louis de Broglie on his 90th Birthday, Edited by S. Diner, D. Fargue, G. Lochak and F. Selleri. D. Reidel Publ. Company, Dordrecht, 1-25 (1970); Accardi L., The probabilistic roots of the quantum mechanical paradoxes. Ibid, 47-55; W. De Muynck and W. De Baere W., Ann. Israel Phys. Soc., 12, 1-22 (1996); W. De Muynck, W. De Baere, H. Marten, Found. of Physics, 24, 1589-1663 (1994); W. De Baere, Lett. Nuovo Cimento, 39, 234-238 (1984); I. Pitowsky, Phys. Rev. Lett, 48, N.10, 1299-1302 (1982); Phys. Rev. D, 27, N.10, 2316-2326 (1983); S.P. Gudder, J. Math Phys., 25, 2397- 2401 (1984); W. Muckenheim, Phys. Reports, 133, 338-401 (1986).

[6]. A. Yu. Khrennikov, Dokl. Akad. Nauk SSSR, ser. Matem., 322, No. 6, 1075-1079 (1992);J. Math. Phys., 32, No. 4, 932-937 (1991); Physics Letters A, 200, 119-223 (1995); Physica A, 215, 577-587 (1995); Int. J. Theor. Phys., 34, 2423-2434 (1995); J. Math. Phys., 36, No.12, 66256632 (1995); A.Yu. Khrennikov, p-adic valued distributions in mathematical physics. Kluwer Academic Publishers, Dordrecht (1994); A.Yu. Khrennikov, 
Non-Archimedean analysis: quantum paradoxes, dynamical systems and biological models. Kluwer Acad.Publ., Dordreht, The Netherlands, 1997.

[7] Kolmogoroff A. N., Grundbegriffe der Wahrscheinlichkeitsrechnung. Springer Verlag, Berlin (1933); reprinted: Foundations of the Probability Theory. Chelsea Publ. Comp., New York (1956).

[8] T. L. Fine, Theories of probabilities, an examination of foundations. Academic Press, New York (1973).

[9] R. von Mises, The mathematical theory of probability and statistics. Academic, London (1964).

[10] P.H. Eberhard, Nuovo Cimento, B, 46, 392-400 (1978); W. de Muynck, J. Steklenborg, Ann. Phys., 45, 222-234(1988).

[11] A. Fine, Phys. Rev. Letters, 48, 291-295 (1982). 